\title{
Investigation of fermentation conditions for Candida bombicola ACTT22214 from molasses and soybean oil for sophorolipid production
}

\author{
Tho P. Le ${ }^{1 *}$, Huong T. T. Le ${ }^{1}$, Hiep M. Dinh ${ }^{2}, \&$ Hue B. T. Nguyen ${ }^{1}$ \\ ${ }^{1}$ Faculty of Biology and Biotechnology, University of Science, Ho Chi Minh City, Vietnam \\ ${ }^{2}$ High-Tech Agriculture Park, Ho Chi Minh City, Vietnam
}

\begin{abstract}
ARTICLE INFO
Research Paper

Received: October 18, 2018

Revised: December 02,2018

Accepted: December 14, 2018
\end{abstract}

Keywords

Antibacterial

Candida bombicola

Fermentation

Sophorolipid

Surfactant

\section{* Corresponding author}

Le Phuoc Tho

Email: phuoctho022010@gmail.com

\begin{abstract}
Sophorolipid (SL) is a biosurfactant belonging to the glycolipids group and was produced by harmless Candida bombicola ACTT22214 and has been widely used in many fields in our life. In order to search for appropriate condition for C. bombicola fermentation producing SL with high efficiency, this study focused on the investigation of the soy oil concentration, molasses concentration, fermentation time, $\mathrm{pH}$ and temperature. SL products were tested for antimicrobial activity, antioxidant, emulsifier, foaming ability. The highest content of SL was $43.27 \pm 0.30 \mathrm{~g} / \mathrm{L}$ under conditions of: soybean oil content $5 \%$, molasses content $150 \mathrm{~g} / \mathrm{L}$, fermentation time 7 days, $\mathrm{pH}=5,28^{0} \mathrm{C}$ fermentation temperature. The antibacterial activity of SL was good: the highest resistance to Candida albicans $(16.33 \pm 1.15 \mathrm{~mm})$, good resistance to Bacillus spizizenii $(13.67 \pm 0.58 \mathrm{~mm})$, resistance to Staphylococcus aureus $(12.67 \pm$ $1.15 \mathrm{~mm}$ ), relatively weak resistance to Pseudomonas aeruginosa $(11.33 \pm 0.58 \mathrm{~mm})$ and Escherichia coli $(9.67 \pm 0.58 \mathrm{~mm})$. The antioxidant capacity of SL was quite high with an $\mathrm{IC}_{50}$ value of $6.024 \mathrm{mg} / \mathrm{mL}$. The emulsifying capacity of SL was equivalent to the emulsification of the tween 20 at a concentration of $5-10 \mathrm{mg} / \mathrm{mL}$. SL had the ability to foam evenly from concentrations of 5 to 20 $\mathrm{mg} / \mathrm{mL}$ but not higher than the corresponding concentrations of tween 20, SL was smooth, even, stable longer than tween 20.
\end{abstract}

Cited as: Le, T. P., Le, H. T. T., Dinh, H. M., \& Nguyen, H. B. T. (2018). Investigation of fermentation conditions for Candida bombicola ACTT22214 from molasses and soybean oil for sophorolipid production. The Journal of Agriculture and Development 17(6), 50-62.

\section{Introduction}

Surfactants are bipolar compounds reducing surface tension between liquids with liquids, solids or gases, therefore they are able to combine and dissolve in water or other liquids. Most used surfactants are originally from petroleum and chemically synthesized, these compounds are environmental hazards because of their low biodegradability and high toxicity when dissolved in water (Bogaert, 2008). Thus, searching for replaceable biological surfactants is a necces- sary strategy. Microbiologically synthesized surfactants, including sophorolipid produced by fermentation of $C$. bombicola are considered due to sophorolipid application and commercialization potential, and significantly because sophorolipid are produced by non-pathogenic and safe $C$. bombicola (Bogaert et al., 2011) which performs high productivity (Dubey et al., 2013).

Sophorolipid are glycolipid biological surfactants, they are bipolar molecules formed by one disaccharide sophorose group bonding hydroxyl group of the second-to-last carbon atom in the 
C16 - C18 fatty acid chain. For the production of sophorolipid by the fermentation of $C$. bombicola, two main substrates are provided: hydrophiliccarbon source: glucose and hydrophobic carbon source: fatty acids, oil, fatty acid ester, alkan. There are two types of sophorolipid: free acid form and lactone ring (Bogaert et al., 2007). This difference results in distinction in sophorolipid physical and chemical characteristics, acidic sophorolipid show foaming ability and high solubility, otherwise, sophorolipid with lactone rings show antibacterial activity and reduce surface tension effectively.

Sophorolipid are used in food, medicine, cosmetics, detergents (Bogaert et al., 2007). Recent research provided some particular applications of SL. Sophorolipid are used in washing powder as a detergent (Gobbert et al., 1984). Sophorolipid emulsification is utilized in petrochemistry. They are used in recovery of secondary petroleum products, removing hydrocarbons in crude petroleum. Sophorolipid are used in treating hydrocarbon contaminated soil and water, absorbing heavy metals in sediment and improve the quality of flour in food industry (Gobbert et al., 1984; Mager et al., 1987; Daniel et al., 1998). Sophorolipid perform antibacterial activity in pimples, dandruff and body's smell treatment, protect skin and hair, stimulate metabolism of epithelial fibroblast cells and synthesis of collagen for skin (Gorin et al., 1961; Cooper \& Paddock, 1984). Sophorolipid also inhibit free radicals and aging elastase enzyme, promoting skin healing and whitening (Isoda et al., 1997). Diacetyl lactone SL can kill carcinoma cell lines such as liver cancer cell line H7402, decrease mortality rate due to septic shock in lab rats (Kim et al., 2005; Daverey \& Pakshirajan, 2009), inhibit the development of leukemia cells (Spencer et al., 1970).

For industrial production and commercialization, SL must be competitive with chemical surfactant in 3 main perspectives: cost, uses and yield. Therefore, searching for low-cost material and setting up procedure for producing SL effectively are important. This study investigates appropriate conditions for C. bombicola fermentation in producing SL from molasses and soybean oil, in addition to conducting test of physical, chemical and biological characteristics of obtained SL.

\section{Material and Methods}

\subsection{Material}

Lyophilized C. bombicola ATCC 22214 was provided by professor Kim Eun-Ki, Inha University, South Korea. Strain was grown in YM Broth (glucose $1 \%$, yeast extract $0.6 \%$, peptone $0.5 \%)$; 2,2-diphenyl-1-picrylhydrazyl (DPPH), 1',4"-sophorolactone 6',6"-diacetate were provided by Sigma (St. Louis, USA). Organic solvent: methanol, ethyl aceate, petroliumether were provided by Xilong company (China). Soy oil content $89.9 \%$ (Simply brand) was produced by Cai Lan vegetable oil company; molasses content $55 \%$ were provided by Kim Minh company; tested bacterial strains were provided by the Research Center of Bioactive Natural Products - University of Science, Ho Chi Minh City.

\subsection{Methods}

\subsubsection{Propagation of Candida bombicola be- fore fermentation}

Lyophilized $C$. bombicola was propagated in YM media, after 48 hours, primary culture was sub cultured, producing secondary culture; $C$. bombicola propagation conditions include: temperature of $28^{0} \mathrm{C}$ and shaking speed at $180 \mathrm{rpm}$ in 48 hours. Secondary culture was used for fermentation and experiments.

\subsubsection{Experiments for investigating appropri- ate conditions for Candida bombicola fermentation producing sophorolipid}

Five one-factor experiments were randomly designed to investigate conditions including: molasses content, soybean oil content, temperature, $\mathrm{pH}$ and fermentation time (sophorolipid obtaining time) (Figure 1). Media used in these experiments contain: yeast extract $0.5 \% ; \mathrm{KH}_{2} \mathrm{PO}_{4}$ $0.1 \% ; \mathrm{MgSO}_{4} .7 \mathrm{H}_{2} \mathrm{O} 0.05 \%, \mathrm{CaCl}_{2} .2 \mathrm{H}_{2} \mathrm{O} 0.01 \%$; $\mathrm{NaCl} 0.01 \%$; peptone $0.07 \%$. These experiments were conducted under conditions of shakingspeed at $180 \mathrm{rpm}$ and fermentation medium was inoculated with $5 \%(\mathrm{v} / \mathrm{v})$ seed medium. Erlenmeyer flasks $(250 \mathrm{~mL})$ with $50 \mathrm{~mL}$ of media were used in the above experiments. Crude sophorolipid yield was observed. 


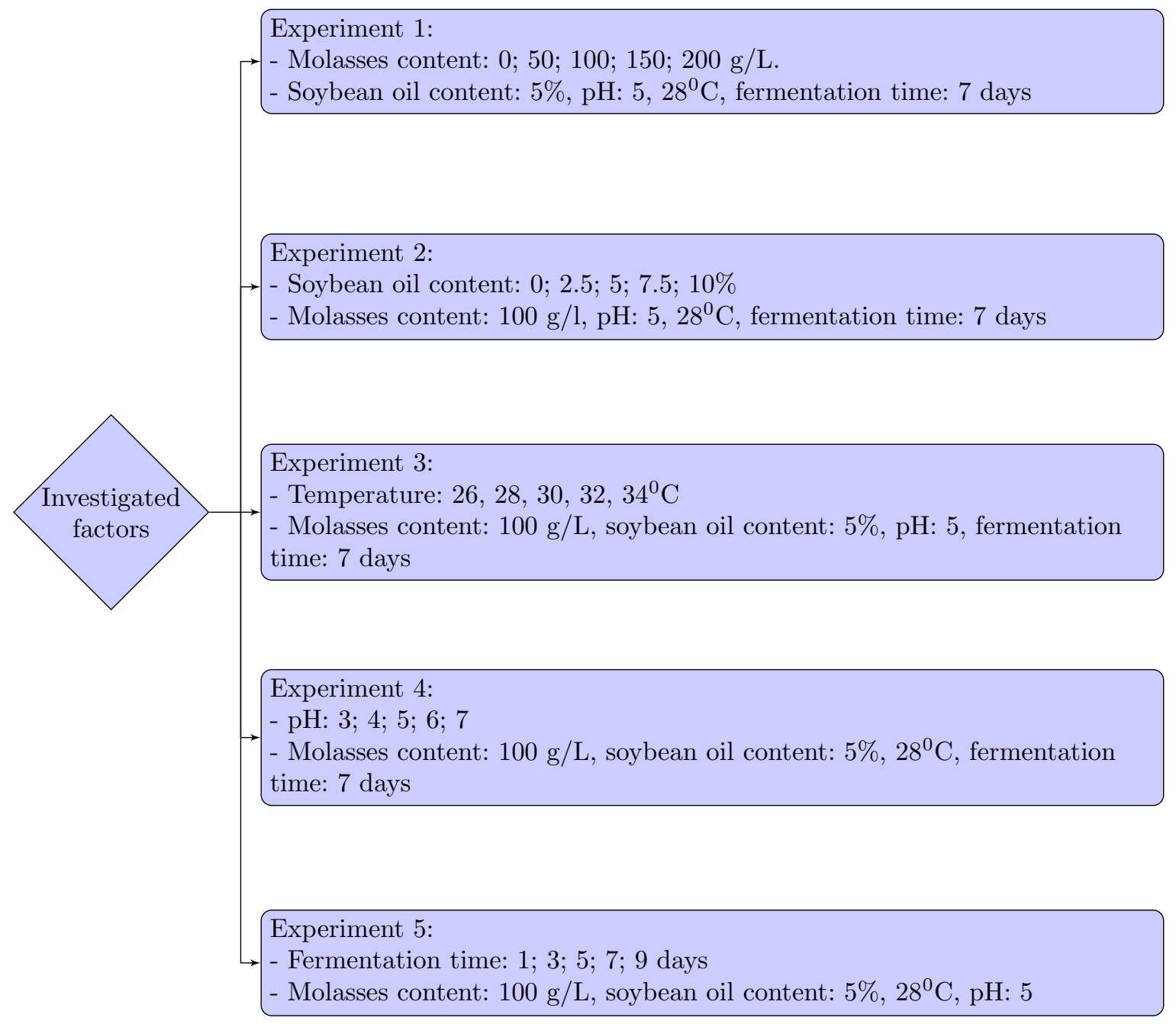

Figure 1. Experiments for investigating appropriate conditions for C. bombicola fermentation producing sophorolipid. 
2.3. Sophorolipid separation from fermented broth and analysis of obtained SL by thin layer chromatogrphy TLC

\subsubsection{Sophorolipid separation from fermented broth}

Ethyl acetate (EtAc) was added into culture broth $(1: 1 \mathrm{v} / \mathrm{v}$, twice), then centrifuged $(6,000$ rpm in 5 minutes), supernatant was collected and vacuum dried in rotary evaporatorat $40^{\circ} \mathrm{C}$ to remove EtAc. After that, Petroliumether: Methanol $(1: 1 \mathrm{v} / \mathrm{v}$, twice) was added and collect the lower layer (crude SL and methanol), then vacuum dried in rotary evaporator at $40^{\circ} \mathrm{C}$ to remove methanol, crude SL was weighed (Figure 2).

\subsubsection{Analysis of obtained SL by thin layer chromatogrphy TLC}

Crude sophorolipid was spotted on chormatography plate then immersed in solvent system containedchloroform:methanol: $\mathrm{H}_{2} \mathrm{O}$ (80:10:2 $\mathrm{v} / \mathrm{v} / \mathrm{v}$ ) in 30 minutes. 1',4"-sophorolactone 6',6"diacetate was used as the standard. After elution, the plate was sprayed with acid sulfuric $90 \%$ and dried at $100^{\circ} \mathrm{C}$, the spots were visualized.

\subsection{Sophorolipid activity analysis}

\subsubsection{Emulsification test}

Sophorolipid was diluted in DMSO 5\% solution into different concentrations then add $5 \mathrm{~mL}$ of each sample into test tubes (diameter: $1.6 \mathrm{~cm}$, height: $16 \mathrm{~cm}$ ). Add the same volume of diesel into these tubes then vortex in 2 minutes, leave for 10 minutes and observe emulsification after 10 minutes, 12 hours and 24 hours, measure the height of emulsified layer, emulsification index after 24 hours was calculated as the formula: E24 $=$ (height of emulsified layer/total height $) \times 100$.

\subsubsection{Foaming ability of SL}

Sophorolipid was diluted in DMSO 5\% solution into different concentrations. After that, add the same volume of $10 \mathrm{~mL}$ of each sample into test tubes (diameter: $1.6 \mathrm{~cm}$, height: $16 \mathrm{~cm}$ ), tight the caps and shake the tubes vertically in 1 minute then leave them for a while. Foaming was observed, and height of foam layers was measured after shaking and after leaving for 5 minutes. Repeat the same procedure or the control (tween
20), the experiment was repeated 3 times.

\subsubsection{Antibacterial activity}

Testing of antibacterial activity was conducted using agar diffusion method. Bacterial strains: Escherichia coli, Staphylococus aureus, Bacillus spizizenii, Pseudomonas aeruginos were inoculated in LB agar plates and Candida albicansin SD agar plates with paper disks were placed on, $20 \mu \mathrm{L} \mathrm{SL}(100 \mathrm{mg} / \mathrm{mL})$ was added to paper disks and incubated $37^{\circ} \mathrm{C}$ in $1-2$ days. Inhibition zones were observed.

\subsubsection{Free radical scavenging activity}

Free radical scavenging activity was determined using DPPH (2,2-diphenyl-1picrylhydrazyl) assay. Crude SL was dissolved in methanol into different concentrations, $100 \mu \mathrm{L}$ of each sample was added into 96-well plate, 100 $\mu \mathrm{L}$ DPPH $300 \mu \mathrm{M}$ was then added and mixed. The plate was incubated at $37^{0} \mathrm{C}$ in 30 minutes, then OD was measured at $517 \mathrm{~nm}$ wavelength. The percentage of free radical scavenging was calculated as the formula: $\%$ antioxidant $=(1-$ OD sample/OD control) x 100.

\section{Results and Discussion}

\subsection{Morphology of C. bombicola}

C. bombicola was recovered and grown in YM media after 2-day inoculation at $28^{\circ} \mathrm{C}$. Culture was diluted into concentration of $10^{-9}$ and inoculated on YM agar plates, the colonies showed creamy color, smooth and glistening dome shape with entire margin, the diameter was from 0.4 $0.7 \mathrm{~cm}$. Observing under microscope using 100X objective lens showed the elongated oval shape of C. bombicola and its budding reproduction (Figure 3).

3.2. Testing of appropriate conditions for $C$. bombicola fermentation producing SL

Recovered $C$. bombicola was grown in YM media in 2 days at $28^{0} \mathrm{C}$, with $180 \mathrm{rpm}$ of shaking speed to obtain the concentration of 109 $\mathrm{CFU} / \mathrm{mL}$. This culture was used as seed medium for below experiments. 


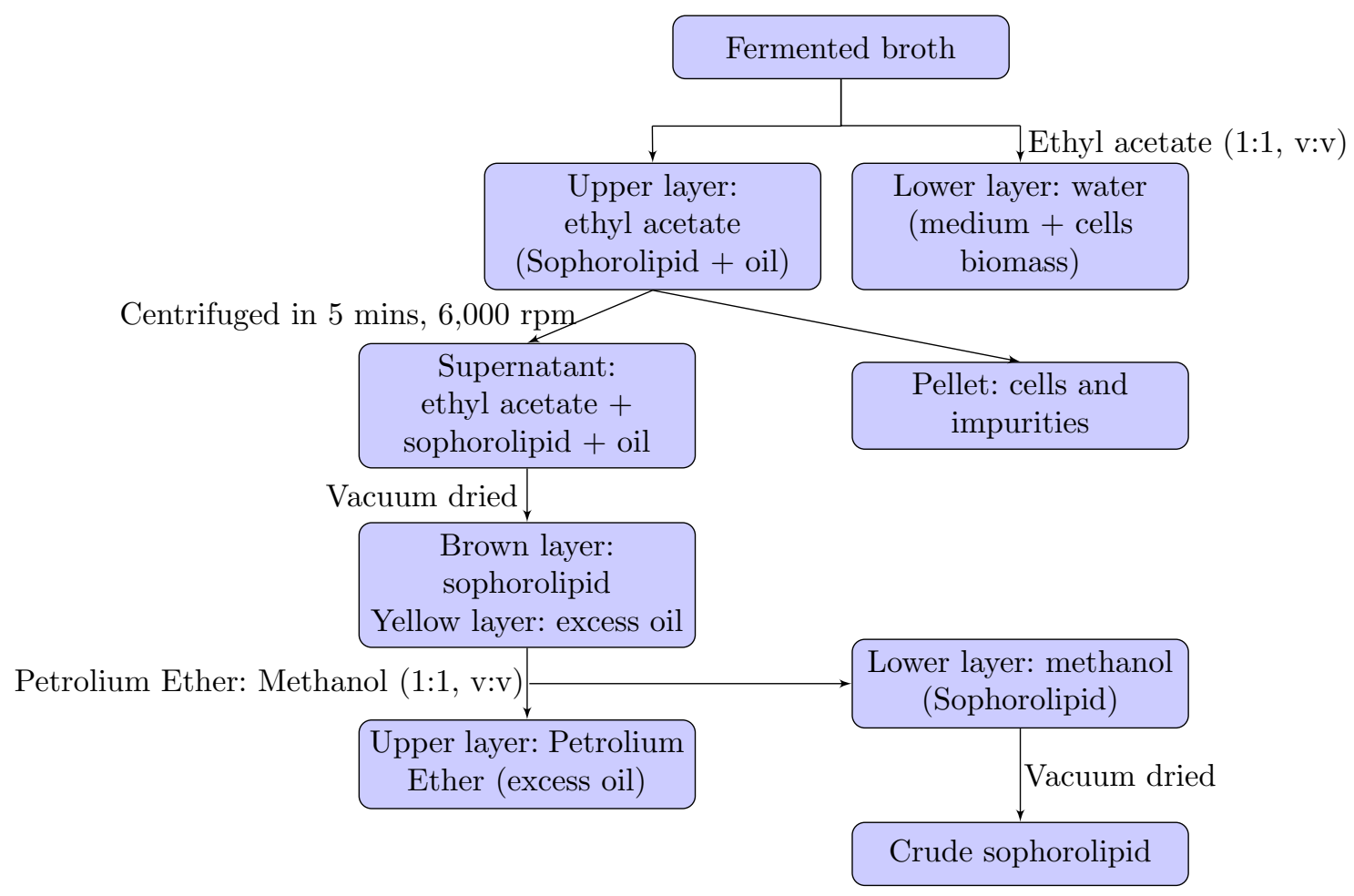

Figure 2. Procedure of separating sophorolipid from fermented broth.
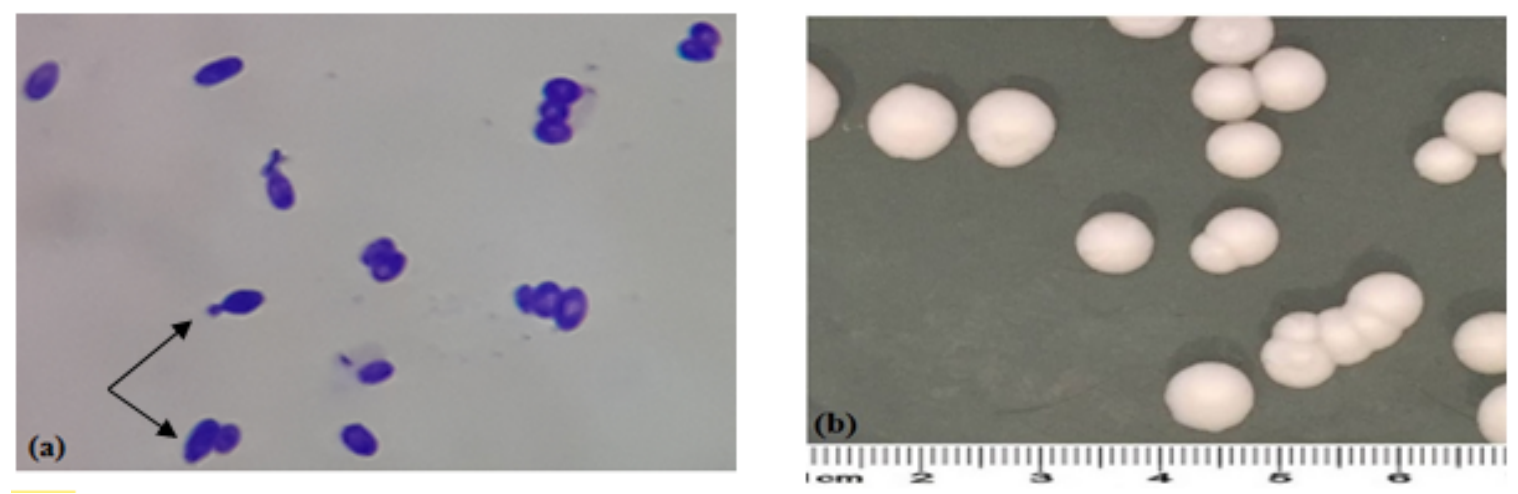

Figure 3. Morphology of yeast C. bombicola. (a) C. bombicola observed under microscope with 100X objective lens shows oval shape and budding reproduction, (b) C. bombicola colonies on YM agar plates after 48-hour incubation at $28^{0} \mathrm{C}$.

3.2.1. Investigation of molasses content affecting the fermentation of $C$. bombicola producing SL

C. bombicola was respectively inoculated into testing samples containing molasses contents of $0 ; 50 ; 100 ; 150,200 \mathrm{~g} / \mathrm{L}$. The result showed that the highest SL production of C. bombicola was $43.27 \pm 0.31 \mathrm{~g} / \mathrm{L}$ when the fermentation me- dia was composed of $150 \mathrm{~g} / \mathrm{L}$. The results were shown the difference from the media composed of $100 \mathrm{~g} / \mathrm{L}$ was not significant. When increasing the molasses concentration to $200 \mathrm{~g} / \mathrm{L}$, SL production decreased. It was probably due to the high osmosis pressure caused by the high concentration. Therefore, the growth and development of C. bombicola were affected in the adapting stage (Table 1). 


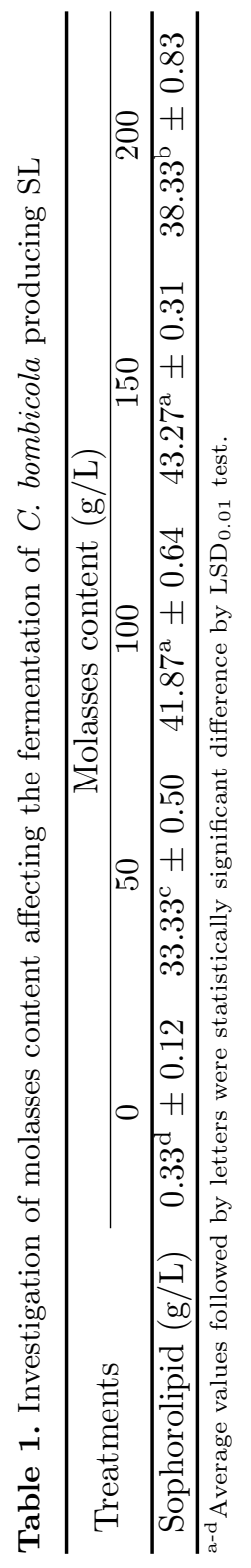

\subsubsection{Investigation of soybean content affect- ing the fermentation of $C$. bombicola producing SL}

C. bombicola was respectively inoculated into testing samples containing soybean contents of 0 ; $2.5 ; 5.0 ; 7.5,10 \%$. The results showed the highest yield of SL was $42.27 \pm 0.31 \mathrm{~g} / \mathrm{L}$ at $5 \%$ of soybean content. However, there was no significant difference from the media containing the soy oil concentration of $7.5 \%$. On the other hand, when increasing the amount of soy oil to $10 \%$, SL production decreased significantly. This was probably due to the lower density of soy oil compared to water and its indissolubility in water. Therefore, the high concentration disrupted the diffusion of oxygen in the media (Table 2).

\subsubsection{Investigation of temperature affecting the fermentation of $C$. bombicola pro- ducing SL}

C. bombicola was respectively inoculated into testing samples for fermentation at the temperature $26 ; 28 ; 30 ; 32 ; 34^{0} \mathrm{C}$. The results showed the highest yield of SL was $41.87 \pm 0.53 \mathrm{~g} / \mathrm{L}$ at the temperature of $28^{\circ} \mathrm{C}$. At $34^{0} \mathrm{C}$, SL yield was nearly none because $C$. bombicola was almost unable to grow so the production of SL was stopped (Table 3).

\subsubsection{Investigation of $\mathrm{pH}$ affecting the fer- mentation of $C$. bombicola producing SL}

C. bombicola was respectively inoculated into testing samples for fermentation at $\mathrm{pH}$ conditions: $3 ; 4 ; 5 ; 6 ; 7$. The results showed the highest yield of SL was $42.07 \pm 0.53 \mathrm{~g} / \mathrm{L}$ at $\mathrm{pH}=5$. We found that there was no significant difference from the result when $\mathrm{pH}=6$ (Table 4 ).

\subsubsection{Investigation of product obtaining time affecting the fermentation of $C$. bombi- cola producing SL}

C. bombicola was respectively inoculated into testing samples for fermentation with obtaining time $1 ; 3 ; 5 ; 7 ; 9$ fermenting days. The result showed the highest SL concentration (41.67 \pm $0,61 \mathrm{~g} / \mathrm{L})$ when the obtaining time was after 7 days. The SL production decreased significantly after 9 days of producing because when the substrate became exhausted, $C$. bombicola would use SL as a nutrient source. Therefore, the in- 

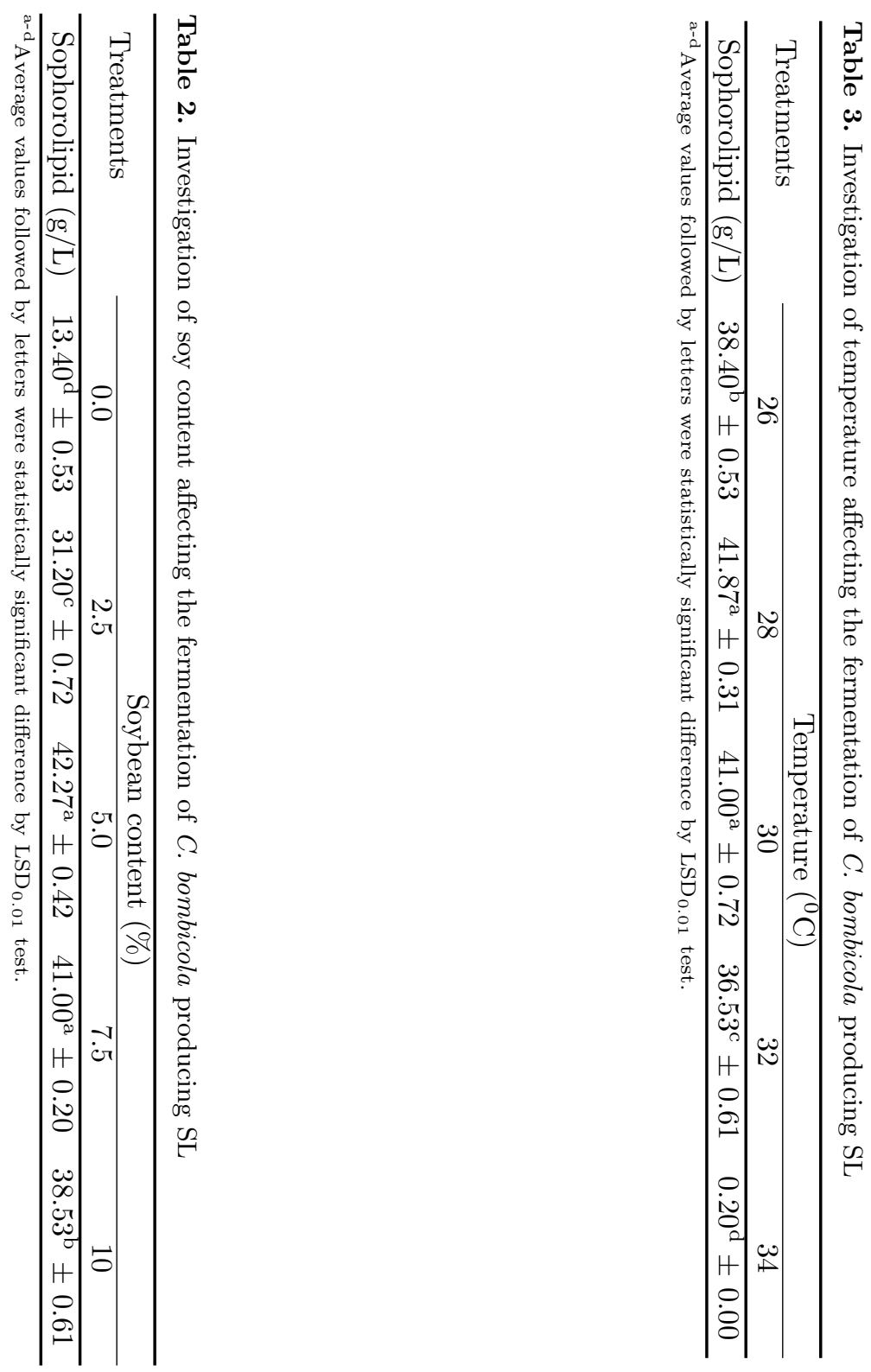


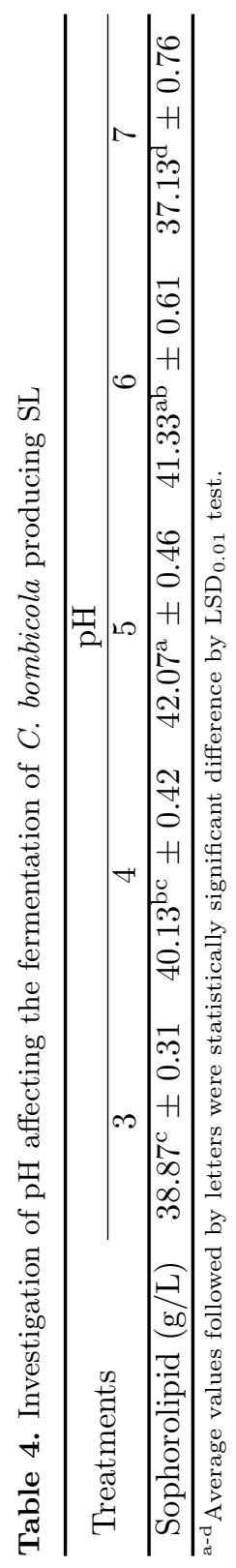

appropriate SL obtaining time decreased the SL amount significantly (Table 5).

Therefore, after the above experiments, the appropriate conditions for C. bombicola fermentation producing SL were shown: $150 \mathrm{~g} / \mathrm{L}$ of molasses content, $5 \%$ of soybean oil content, $28^{0} \mathrm{C}$, $\mathrm{pH}=5$, obtaining time is after 7 days of fermentation. From the above data, fermentation of C. bombicola was conducted, the yield was 43.27 $\pm 0.30 \mathrm{~g} / \mathrm{L}$. SL obtained is brown,viscous liquid (Figure 4).

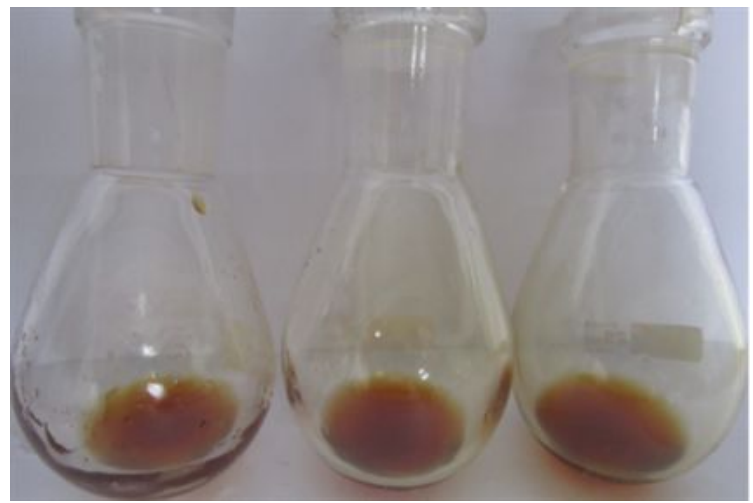

Figure 4. Obtained SL from the fermentaion of $C$. bombicola.

According to Table 6, SL yield was considered high $(43.27 \mathrm{~g} / \mathrm{L})$, higher than results in the research of Asmer et al. (1988), Nguyen et al. (2017) and Le et al. (2016) which were 34; 21.8 and 14.6 $\mathrm{g} / \mathrm{L}$, respectively. However, SL yield was lower than in research of Cooper \& Paddock (1984), Deshpande \& Daniels (1995), and Zhou et al. (1992) which were 68; 97 and $138 \mathrm{~g} / \mathrm{L}$, respectively. The difference in SL yield can be due to different source of substrates, fermenting conditions as well as the time of obtaining SL. Therefore, it is important to have further research on optimal conditions as well as appropriate substrate contents for the fermentation.

\subsection{Analysis of obtained SL by thin layer chromatogrphy TLC}

Figure 5 shows the existance of 1',4"'- sophorolactone 6',6"-diacetate in crude SL, proving the suitability of crude SL extraction in obtaining SL from fermented broth. In addition, visualized spots were at different positions showing different structures of obtained SL. 

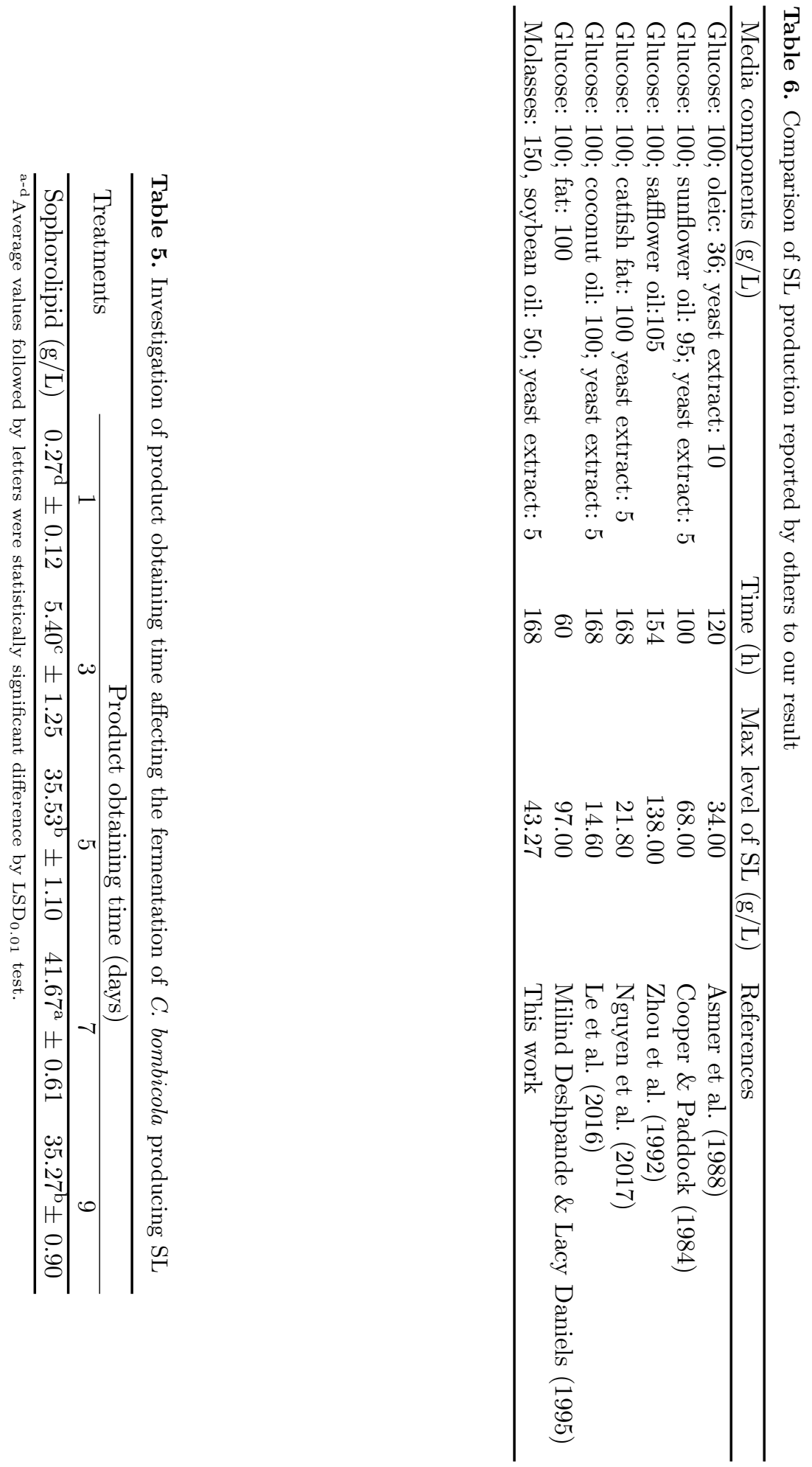


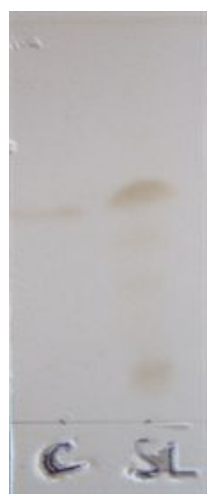

Figure 5. Chromatogram of SL analysis obtained SL; (C) standard substance (1',4"-sophorolactone 6',6"-diacetate), the $\mathrm{Rf}$ value is 0.55 .

\subsection{Testing of physical, chemical and biologi- cal of SL}

\subsubsection{Foaming ability of SL}

To determine the foaming ability of SL, shake test tubes containing SL and 20 tween (control) dilutled in DMSO 5\% solution into different concentrations were shaked vertically in 1 minute, observing and measure the height of foam layer twice: after shaking and after leaving for 5 minutes.

Based on Figure 6, the foaming ability of the two surfactants SL and tween 20 increased as their concentrations increased. The amount of foam produced by tween 20 was much higher than by SL. After 5 minutes, the amount of foam formed by the two surfactants was reduced but negligible, the foam volume was maintained quite well. At the same time, smooth, uniform, and stable foam produced by SL was formed, relatively durable compared to the tween 20 .

\subsubsection{Testing the emulsifying ability of SL}

Determination of emulsifying ability of SL was carried out by uniformly vortexing SL and tween 20 solutions diluted in 5\% DMSO solution into different concentrations in 2 minutes, leave them to stand still and observe the emulsion. After 10 minutes, 12 hours and after 24 hours, measure the height of the emulsified layer, determine the E24 index of solutions, with E24 being the emulsifying percentage after 24 hours.

Based on Figure 7, the emulsification of both SL and tween 20 increased as the concentration increased. However, at the concentration of 20 $\mathrm{mg} / \mathrm{mL}$, the emulsification of SL is very low, almost none; while at concentrations of $5 \mathrm{mg} / \mathrm{mL}$ and $10 \mathrm{mg} / \mathrm{mL}$, the emulsification of SL is almost equivalent to tween 20 . The decrease or loss of emulsification capacity when the concentration of SL increases is due to SL's tendency of aggregate into large masses, its contact and dispersion into the water phase and the oil phase decreases, sometimes the accumulation was too much, the SL mass becomes heavier, leading to deposition to the bottom and making the emulsification not good.

\subsubsection{Examination of antibacterial activity of SL}

In order to determine the antibacterial activity of SL, aspirate $20 \mu \mathrm{L}$ of $100 \mathrm{mg} / \mathrm{mL}$ SL solution onto paper plates placed on the surface of agar containing the tested microorganisms. Positive antibiotics are gentamicin $(100 \mu \mathrm{g} / \mathrm{ml})$ or terbinafine $(100 \mu \mathrm{g} / \mathrm{mL})$ and negative methanol $(90 \%)$.

Based on Figure 8 and Table 7, it shows that SL is most resistant to $C$. albicans, followed by B. spizizenii, then $S$. aureus, and finally weak resistance to $P$. aeruginosa and E. coli. Thus, it is possible to conclude that SL is more resistant to Gram (+) bacteria than Gram (-) bacteria. This finding is consistent with researches the research of Kim et al. (2005). The resistance to C. albicans which is a fungus parasitic on human body of SL proves that SL has the potential to be used as safe detergent and antiseptic.

Table 7. Results of the ring diameter of sophorolipid resistance for microorganisms

\begin{tabular}{lc}
\hline Microorganisms & $\begin{array}{c}\text { Antibacterial ring } \\
\text { diameter of SL }(\mathrm{mm})\end{array}$ \\
\hline B. spizizenii & $13.68 \pm 0.58$ \\
C. albicans & $16.33 \pm 1.15$ \\
E. coli & $09.67 \pm 0.58$ \\
P. aeruginosa & $11.33 \pm 0.58$ \\
S. aureus & $12.67 \pm 1.15$ \\
\hline
\end{tabular}

3.4.4. Investigation of the antioxidant capacity of sophorolipid by DPPH (1,1Diphenyl-2-picrylhydrazyl)

Add $100 \mu \mathrm{l}$ of each SL solutions at different concentrations into 96 well microplates. Af- 

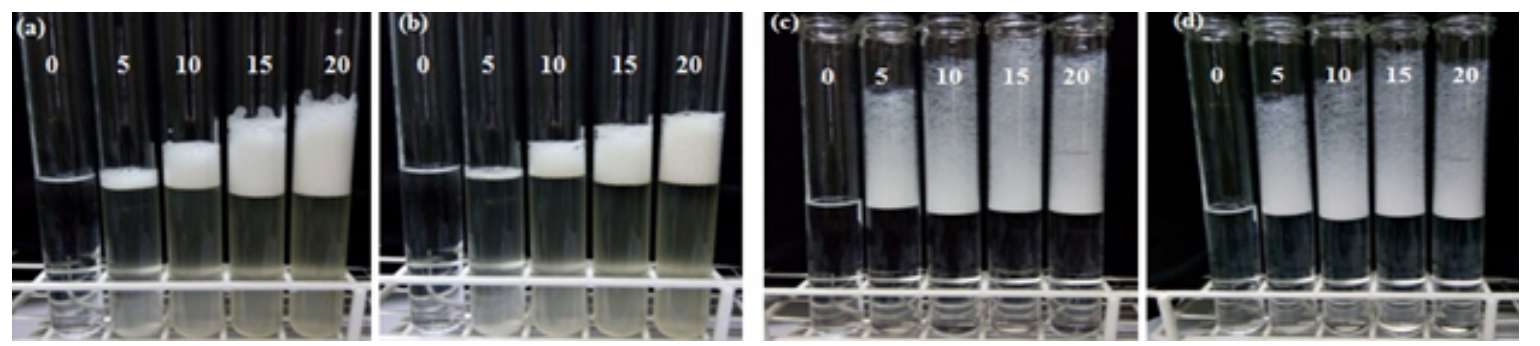

Figure 6. Results of the foaming test of SL and tween 20. (a) SL after shaking, (b) SL after leaving for 5 minutes, (c) Tween 20 after shaking, (d) Tween 20 after leaving for 5 minutes. SL/tween 20 concentrations were $0 ; 5 ; 10 ; 15 ; 20(\mathrm{mg} / \mathrm{mL})$.
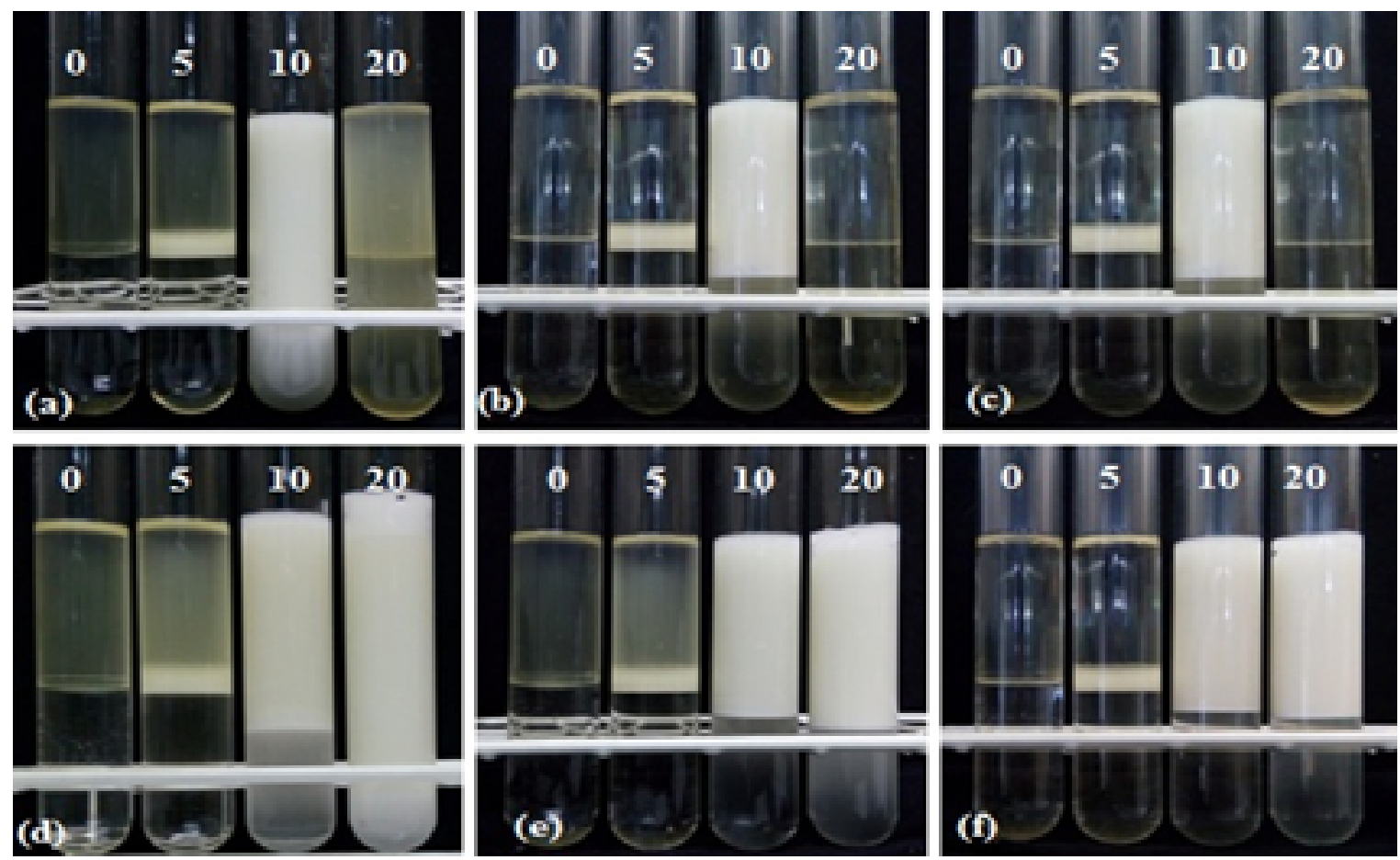

Figure 7. Results from emulsion investigations of SL and tween 20. (a), (b), (c) emulsification of SL after 20 minutes, 12 hours and 24 hours; (d), (e), (f) emulsification of tween 20 after 20 minutes, 12 hours and 24 hours. The investigated SL/tween 20 concentrations were $0 ; 5 ; 10 ; 20(\mathrm{mg} / \mathrm{mL})$.

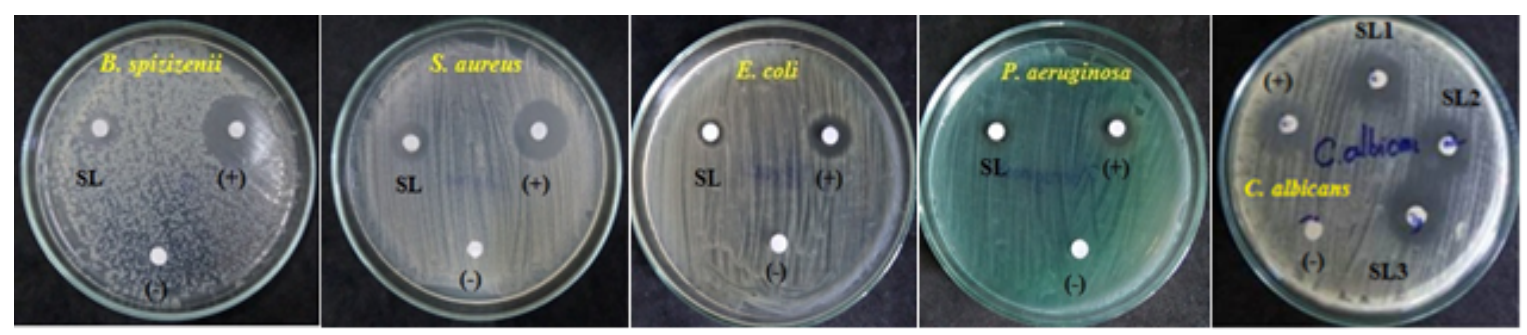

Figure 8. Antibacterial results of SL with paper disk method, (+): Positive test; (-): Negative test. 
ter adding $100 \mu \mathrm{l} \mathrm{DPPH}$, incubate at $37^{0} \mathrm{C}$ for 30 minutes, then determine the color by ELISA reader and acknowledge the percentage of antioxidant corresponding to each concentration. Therefore, determination of the expressed curve shows the relationship between the substance concentration and the corresponding antioxidant percentage of SL.

From Figure 9, the $\mathrm{IC}_{50}$ value is $6.024 \mathrm{mg} / \mathrm{mL}$. With $\mathrm{IC}_{50}$ value of $6.024 \mathrm{mg} / \mathrm{mL}$, it can be seen that the antioxidant capacity of SL is quite good. This can be applied to produce useful antioxidants in cosmetic products such as skin lotion, aiming to care and protect the skin.

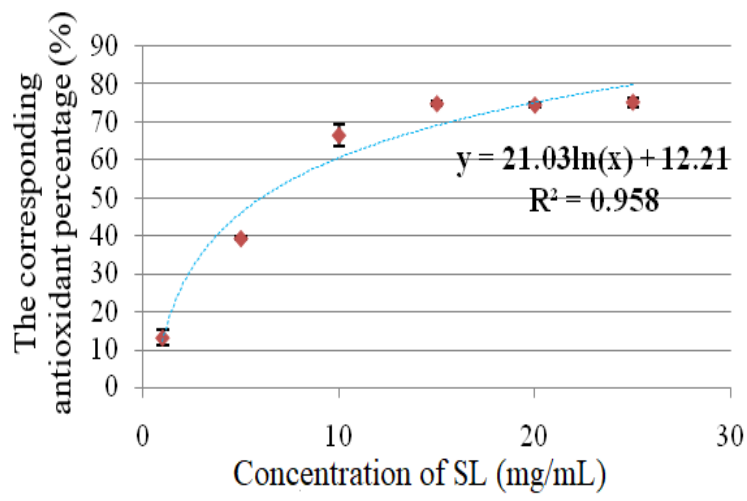

Figure 9. The graph shows the antioxidant percentages of SL by DPPH.

\section{Conclusion}

Initially, suitable conditions for fermentation of C. bombicola for SL production are found to be quite high at $43.27 \mathrm{~g} / \mathrm{L}$ with the following parameters: $150 \mathrm{~g} / \mathrm{L}$ of molasses; $5 \%$ of soybean oil, fermentation temperature is $28^{0} \mathrm{C}, \mathrm{pH}=5$, fermentation time is 7 days. SL product has good antimicrobial properties, antioxidant capacity with an $\mathrm{IC}_{50}$ of $6.024 \mathrm{mg} / \mathrm{mL}$, the ability to foam, durable and stable emulsification equivalent to chemical surfactants. Therefore, it shows the high application potential of SL in this study in areas such as cosmetics and detergents.

\section{Acknowledgements}

The study was funded by the Ho Chi Minh City Department of Science and Technology.

\section{References}

Asmer, H., Lang, S., Wagner, F., \& Wray, V. (1988). Microbial production, structure elucidation and bioconversion of sophorose lipids. Journal of the American Oil Chemists' Society 65(9), 1460-1466.

Bogaert, I. N. V. (2008). Literature review on microbial production and application of the biosurfactant sophorolipids, microbial synthesis of sophorolipids by the yeast Candida bombicola. (Unpublished doctoral dissertation). University of Ghent, Ghent, Belgium.

Bogaert, I. N. V., Zhang, J., \& Soetaert, W. (2011). Microbial synthesis of sophorolipids. Process Biochemistry 46(4), 821-833.

Bogaert, I. N. V., Saerens, K., de Muynck, C., Develter, D., Soetaert, W., \& Vandamme, E. J. (2007). Microbial production and application of sophorolipids. Applied Microbiology and Biotechnology 76(1), 23-34.

Cooper, D. G., \& Paddock, D. A. (1984). Production of a biosurfactant from Torulopsis bombicola. Applied and Environmental Microbiology 47(1), 173-176.

Daniel, H. J., Reuss, M., \& Syldatk, C. (1998). Production of sophorolipids in high concentration from deproteinized whey and rapeseed oil in a two stage fed batch process using Candida bombicola ATCC 22214 and Cryptococcus curvatus ATCC 20509. Biotechnology Letters 20(12), 1153-1156.

Daverey, A., \& Pakshirajan, K. (2009). Production, characterization, and properties of sophorolipids from the yeast Candida bombicola using a low-cost fermentative medium. Applied Biochemistry and Biotechnology 158(3), 663-674.

Deshpande, M., \& Daniels, L. (1995). Evaluation of sophorolipid biosurfactant production by Candida bombicola using animal fat. Bioresource Technology 54(2), 143-150.

Dubey, P., Selvaraj, K., \& Prabhune, A. (2013). Sophorolipids: in self assembly and nanomaterial synthesis. World Journal of Pharmacy and Pharmaceutical Sciences 2(3), 1107-1133.

Gobbert, U., Lang, S., \& Wagner, F. (1984). Sophorose lipid formation by resting cells of Torulopsis bombicola. Biotechnology Letters 6(4), 225-230.

Gorin, P. A. J., Spencer, J. F. T., \& Tulloch, A. P. (1961). Hydroxy fatty acid glycosides of sophorose from Torulopsis magnolia. Canadian Journal of Chemistry 39(4), 846- 855 .

Isoda, H., Kitamoto, D., Shinmoto, H., Matsumura, M., \& Nakahara, T. (1997). Microbial extracellular glycolipid induction of differentiation and inhibition of the protein kinase $\mathrm{C}$ activity of human promyelocytic leukemia cell line HL60. Bioscience, Biotechnology and Biochemistry 61(4), 609-614.

Kim, H. S., Kim, Y. B., Lee, B. S., \& Kim, E. K. (2005). Sophorolipid production by Candida bombicola ATCC 22214 from a corn-oil processing byproduct. Journal of Microbiology and Biotechnology 15(1), 55-58. 
Le, L. Q., Ngo, D. D., Hoang, K. Q., Nguyen, D. H., Nguyen, H. L. H., \& Nguyen, H. T. B. (2016). Production and characterization of sophorolipids produced by Candida bombicola from coconut oil. Science \& Technology Development Journal 19(5), 15-25.

Mager, H., Röthlisberger, R., \& Wzagner, F. (1987). Use of sophorolse-lipid lactone for the treatment of dandruffs and body odeur. European patent, 0209783.

Nguyen, H. L. H., Le, L. Q., Vo, S. T., Le, M. V., Le, D. V., Phung, H. T. T., Pham, D. V., \& Nguyen, D. H. (2017). Production and characterization of sophorolipids by Candida bombicola using catfish fat. Ho Chi Minh City University of Education Journal of Science 14(9), 152-159.
Spencer, J. F. T., Gorin, P. A. J., \& Tulloch, A. P. (1970). Torulopsis bombicola sp. Antonie Van Leeuwenhoek 36(1), 129-133.

Zhou, Q. H., Klekner, V., \& Kosaric, N. (1992). Production of sophorose lipids by Torulopsis bombicola from safflower oil and glucose. Journal of the American Oil Chemists' Society 69(1), 89-91. 